\title{
The bends in the slopes of radial abundance gradients in the disks of spiral galaxies - Do they exist?
}

\author{
L. S. Pilyugin ${ }^{\star}$
}

\begin{abstract}
Main Astronomical Observatory of National Academy of Sciences of Ukraine, 27 Zabolotnogo str., 03680 Kiev, Ukraine
Received 14 March 2002 / Accepted 1 October 2002

Abstract. Spiral galaxies with a reported bend in the slope of gradient in the oxygen abundances $(\mathrm{O} / \mathrm{H})_{R_{23}}$, derived with traditionally used $R_{23}$-method, were examined. It is shown that the artificial origin of the reported bends can be naturally explained. Two situations that result in a false bend in the slope of $(\mathrm{O} / \mathrm{H})_{R_{23}}$ gradient are indicated. It is concluded that at the present time there is no example of a galaxy with an undisputable established bend in the slope of the oxygen abundance gradient.
\end{abstract}

Key words. galaxies: abundances - galaxies: ISM - galaxies: spiral

\section{Introduction}

It has been known for a long time (Searle 1971; Smith 1975) that disks of spiral galaxies can show radial oxygen abundance gradients, in the sense that the oxygen abundance is higher at the central part of disk and decreases with galactocentric distance. By now, spectra have been obtained for hundreds of $\mathrm{H}$ II regions in disks of spiral galaxies. The characteristic oxygen abundances (the oxygen abundance at a predetermined galactocentric distance) and radial oxygen abundance gradients were obtained for a large sample of spiral galaxies (Vila-Costas \& Edmunds 1992; Zaritsky et al. 1994; van Zee et al. 1998, among others). It was obtained that nearly all the gradients are reasonably well fitted by a single exponential profile, although in several cases the gradient slope may not be constant across the disk but instead flattens (or steepens) in the outer disk.

Zaritsky (1992) has hypothesized that the oxygen abundance gradients in the disks of spiral galaxies flatten noticeably at the radius where the rotation curve changes from linearly rising to flat, and he has suggested a star-forming viscous disk model of galaxy evolution to argue in favour of his hypotheses. Friedli et al. (1994) and Friedli \& Benz (1995) have predicted breaks in the slope of abundance gradients in spiral galaxies with young bars $(<0.5 \div 1 \mathrm{Gyr})$. The break in the slope of abundance gradient in spiral galaxy then was considered as an indicator of a recently formed bar in that galaxy (Roy \& Walsh 1997; Friedli 1999; Considére et al. 2000).

The signs of a break in the slope of abundance gradients were found on the basis of oxygen abundances derived with the abundance indicator $R_{23}$ or/and $R_{3}\left(R_{23}\right.$-method) (Vilchez et al. 1988; Zaritsky 1992; Vila-Costas \& Edmunds 1992; Zaritsky et al. 1994; Roy \& Walsh 1997). Zaritsky (1992) and Zaritsky et al. (1994) noted that the bend in the abundance indicator $R_{23}$ (or $R_{3}$ ) gradient may not reflect a corresponding bend in

\footnotetext{
^ e-mail: pilyugin@mao.kiev.ua
}

the abundance gradient. Recently it has been shown (Pilyugin $2000,2001 \mathrm{a}, \mathrm{b})$ that the oxygen abundance derived with the $R_{23}$-method involves a systematic error depending on the excitation parameter $P$ : the $R_{23}$-method provides more or less realistic oxygen abundances in high-excitation $\mathrm{H}$ II regions and yields an overestimated oxygen abundances in low-excitation $\mathrm{H}$ II regions. This is in agreement with the result of Kinkel \& Rosa (1994), who showed the need to lower all $\mathrm{H}_{\text {II }}$ region abundances obtained on the basis of the $R_{23}$ calibration after Edmunds \& Pagel (1984) at intrinsic solar-like O/H values and above. Castellanos et al. (2002) also found that the $R_{23}$-method yields an overestimated oxygen abundance in low-excitation $\mathrm{H}$ II regions. A new way of oxygen abundance determination in $\mathrm{H}_{\text {II }}$ regions ( $P$-method) was suggested (Pilyugin 2001a). It was demonstrated that the oxygen abundances derived with the $P$-method are as credible as ones derived with the classic $T_{\mathrm{e}^{-}}$ method (Pilyugin 2001a,b). It should be noted, however, that the $P$-method is established on the basis of $\mathrm{H}_{\text {II }}$ regions with $R_{23}$ larger than 2 and the validity of this method in the case of $\mathrm{H}$ II regions with $R_{23}$ less than 2 (most metal-rich $\mathrm{H}$ II regions) may be disputed.

The radial distribution of oxygen abundances derived through the $P$-method in some galaxies with a reported bend in the slope of abundance will be considered here to examine the reality of the reported bends.

\section{Galaxies with a reported bend in the slope of the abundance gradient}

\subsection{Preliminary remarks}

The following relationship between oxygen abundance and strong line intensities was suggested in Pilyugin (2001a)

$12+\log (\mathrm{O} / \mathrm{H})_{P}=\frac{R_{23}+54.2+59.45 P+7.31 P^{2}}{6.07+6.71 P+0.37 P^{2}+0.243 R_{23}}$ 
where $R_{23}=R_{2}+R_{3}, R_{2}=I_{[\mathrm{OII}] \lambda 3727+\lambda 3729} / I_{\mathrm{H} \beta}, R_{3}=$

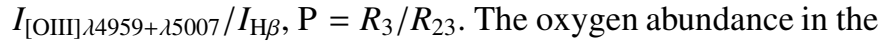
$\mathrm{H}$ II region derived with Eq. (1) will be referred to as $(\mathrm{O} / \mathrm{H})_{P}$. The relationship between oxygen abundance and strong line intensities has two values with two distinctive parts named usually the lower and upper branches of the $R_{23}-\mathrm{O} / \mathrm{H}$ diagram, and so one has to know in advance on which branch of the $R_{23}-\mathrm{O} / \mathrm{H}$ diagram the $\mathrm{H}$ II region lies. The above expression for the oxygen abundance determination in $\mathrm{H}_{\text {II }}$ regions, Eq. (1), is valid for $\mathrm{H}$ II regions with $12+\log (\mathrm{O} / \mathrm{H})$ higher than around 8.2.

The $\mathrm{O} / \mathrm{H}-R_{23}$ calibration after Edmunds \& Pagel (1984) will be used here for the oxygen abundance determination with the $R_{23}$-method. For the sake of convenience, their calibration for the upper branch of the $R_{23}-\mathrm{O} / \mathrm{H}$ diagram was approximated by the polynomial

$$
\begin{aligned}
12+\log (\mathrm{O} / \mathrm{H})_{R_{23}}= & 9.302-0.403 x-0.675 x^{2} \\
& -0.701 x^{3}+0.666 x^{4}
\end{aligned}
$$

where $x=\log R_{23}$. The oxygen abundance in $\mathrm{H}$ II region derived with Eq. (2) will be referred to as $(\mathrm{O} / \mathrm{H})_{R_{23}}$.

\subsection{False bends of the "first type"}

We will start from a consideration of the well-observed galaxy M101. The break in the slope of the abundance gradient in M101 was reported by Zaritsky (1992) and by Vila-Costas \& Edmunds (1992). It should be noted that this conclusion was not confirmed by Henry \& Howard (1995), who determined oxygen abundance using a sequential photoionization model analysis procedure and found that the observed behavior of line ratios accross the disk of M101 is consistent with an abundance gradient that is exponential in form and has a constant slope. About ninety measurements of the strong oxygen lines intensities in $\mathrm{H}$ II regions of the galaxy M101 were published (Garnett \& Kennicutt 1994; Garnett et al. 1999; Kennicutt \& Garnett 1996; Kinkel \& Rosa 1994; McCall et al. 1985; Rayo et al. 1982; Searle 1971; Shields \& Searle 1978; Smith 1975; Torres-Peimbert et al. 1989; van Zee et al. 1998).

Figure 1a shows the radial $(\mathrm{O} / \mathrm{H})_{R_{23}}$ abundance distribution (the triangles) for the $\mathrm{H}$ II regions in M101 derived with Eq. (2). The galactocentric distances are normalized to the isophotal radius $R_{25}$ which was taken to be equal to $14.42 \mathrm{arcmin}$ (de Vaucouleurs et al. 1991). Figure $1 \mathrm{~b}$ shows the $\operatorname{radial}(\mathrm{O} / \mathrm{H})_{P}$ abundance distribution (the filled circles) for $\mathrm{H}$ II regions in M101 derived with Eq. (1) using the same values of the strong oxygen lines intensities. As can be seen in Figs. 1a,b there is break in the slope of $(\mathrm{O} / \mathrm{H})_{R_{23}}$ abundance distribution as in the slope of $(\mathrm{O} / \mathrm{H})_{P}$ abundance distribution, at the galactocentric distance $R^{*} \sim 0.6 R_{25}$. Close examination of Figs. 1a,b shows that both the $(\mathrm{O} / \mathrm{H})_{R_{23}}$ and the $(\mathrm{O} / \mathrm{H})_{P}$ distributions in the disk of M101 flatten at the radius $R^{*}$ where the oxygen abundance decreases to around $12+\log (\mathrm{O} / \mathrm{H})=8.2$. The oxygen abundances in $\mathrm{H}$ II regions with galactocentric distances larger than $R^{*}$ are expected to be less than $12+\log (\mathrm{O} / \mathrm{H})=8.2$, i.e they do not belong to the upper branch of the $R_{23}-\mathrm{O} / \mathrm{H}$ diagram. But Eqs. (1) and (2) can be used for oxygen abundance determination in $\mathrm{H}$ II regions of the upper branch of the
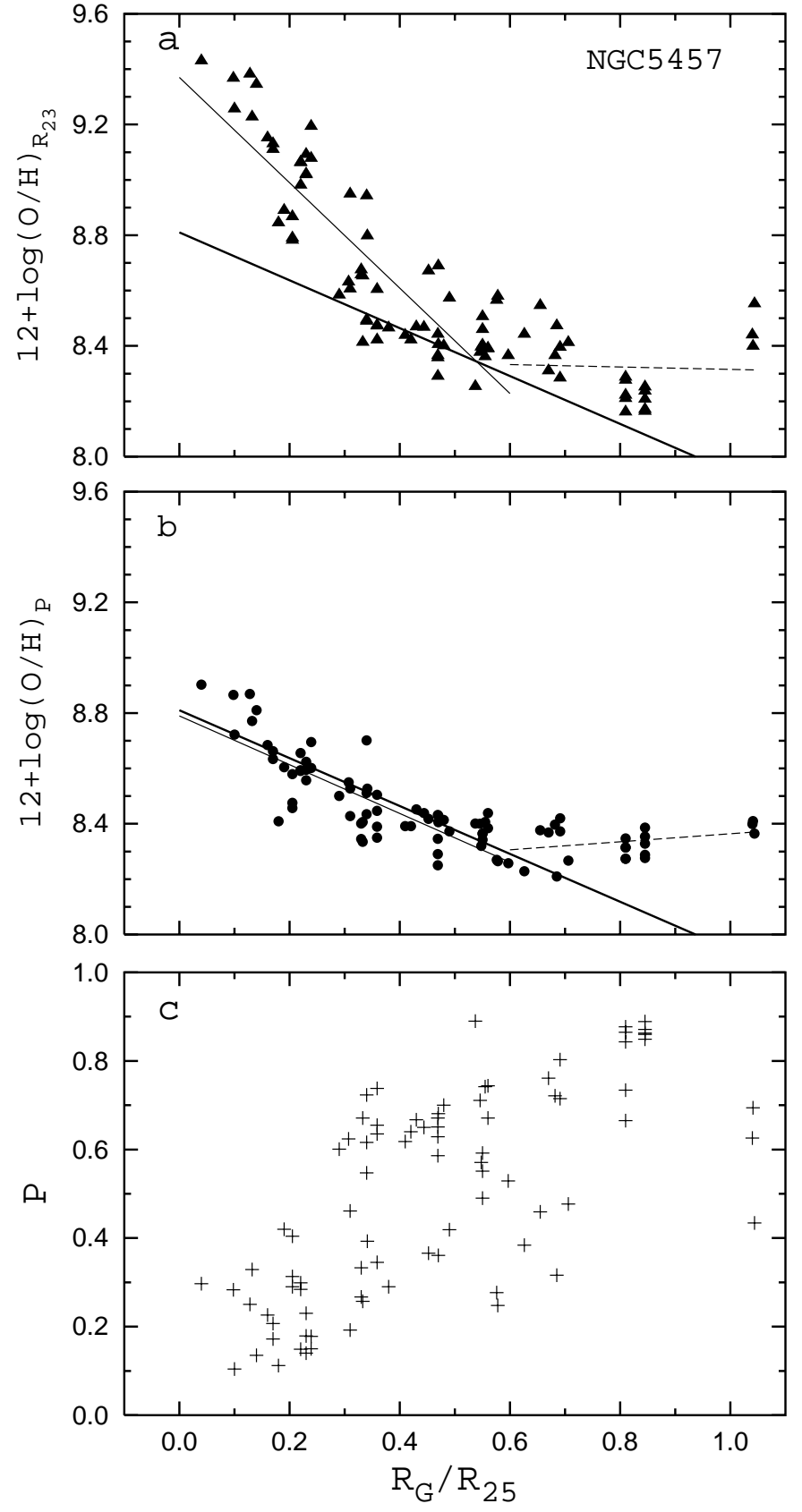

Fig. 1. Gradients in the properties of M101. The galactocentric distances are normalized to the isophotal radius $R_{25}$. a) The triangles are $(\mathrm{O} / \mathrm{H})_{R_{23}}$ abundances in $\mathrm{H}$ II regions derived with Eq. (2). The thin solid line is the best fit to the $\mathrm{H}$ II regions with galactocentric distances less than $0.6 R_{25}$, the dashed line is the best fit to the $\mathrm{H}_{\text {II }}$ regions with galactocentric distances larger than $0.6 R_{25}$. The thick solid line is the the $(\mathrm{O} / \mathrm{H})_{T_{\mathrm{e}}}-R_{\mathrm{G}}$ relation obtained in Pilyugin (2001b). b) The filled circles are $(\mathrm{O} / \mathrm{H})_{P}$ abundances in the same $\mathrm{H}_{\text {II }}$ regions derived with Eq. (1). The thin solid line is the best fit to to $\mathrm{H}_{\text {II }}$ regions with galactocentric distances less than $0.6 R_{25}$, the dashed line is the best fit to $\mathrm{H}_{\text {II }}$ regions with galactocentric distances larger than $0.6 R_{25}$. The thick solid line is the the $(\mathrm{O} / \mathrm{H})_{T_{\mathrm{e}}}-R_{\mathrm{G}}$ relation. c) The excitation parameter $P$ as a function of galactocentric distance.

$R_{23}-\mathrm{O} / \mathrm{H}$ diagram only. Then one can conclude that the use of these equations for the oxygen abundance determination in the $\mathrm{H}$ II regions of M101 with galactocentric distances larger 

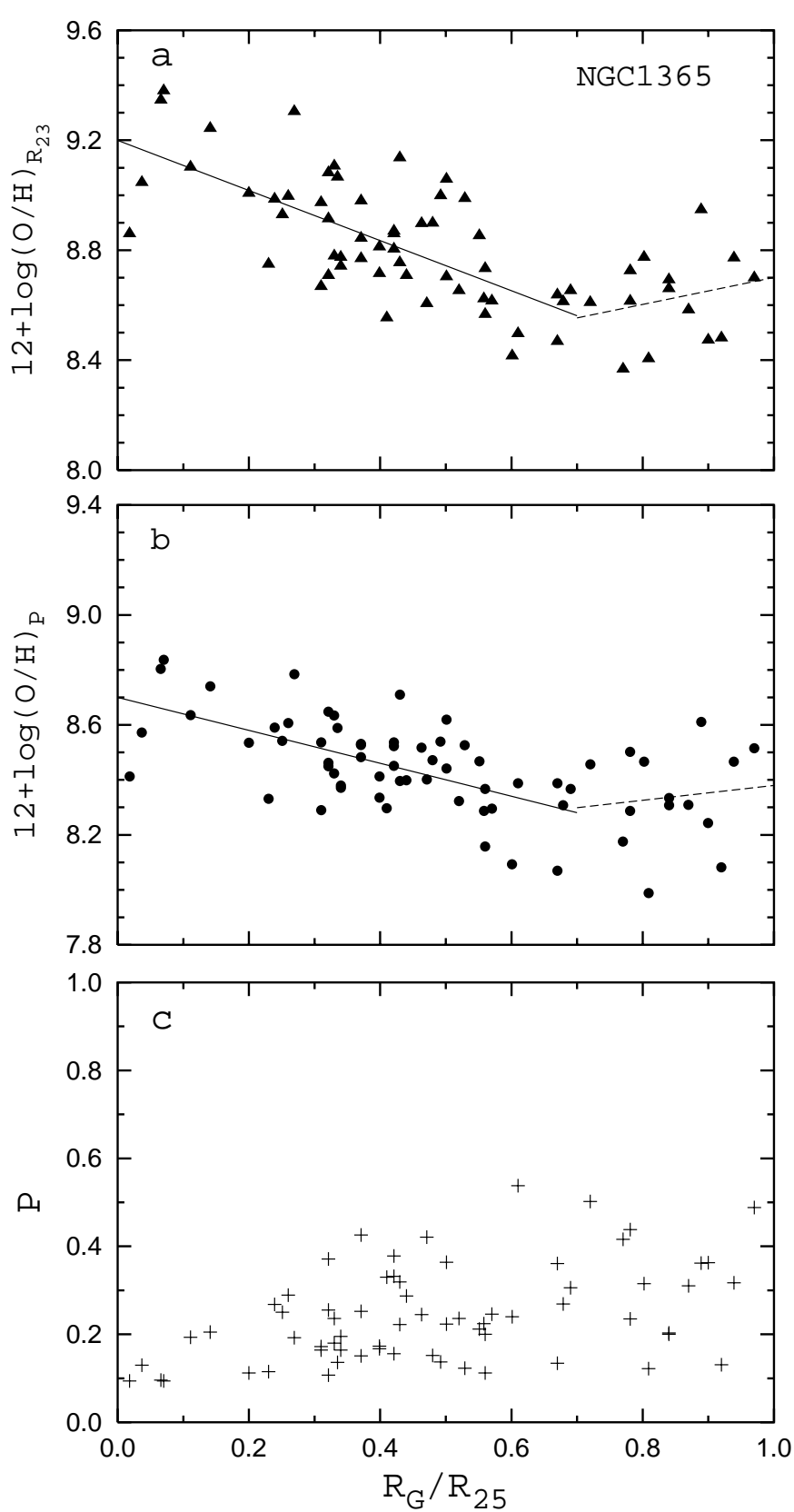

Fig. 2. Gradients in the properties of NGC 1365. The galactocentric distances are normalized to the isophotal radius $R_{25}$. a) The triangles are $(\mathrm{O} / \mathrm{H})_{R_{23}}$ abundances in $\mathrm{H}$ II regions derived with Eq. (2). The thin solid line is the best fit to the $\mathrm{H}$ II regions with galactocentric distances less than $0.7 R_{25}$, the dashed line is the best fit to the $\mathrm{H}$ II regions with galactocentric distances larger than $0.7 R_{25}$. b) The filled circles are $(\mathrm{O} / \mathrm{H})_{P}$ abundances in the same $\mathrm{H}$ II regions derived with Eq. (1). The thin solid line is the best fit to to $\mathrm{H}_{\text {II }}$ regions with galactocentric distances less than $0.7 R_{25}$, the dashed line is the best fit to $\mathrm{H}_{\text {II }}$ regions with galactocentric distances larger than $0.7 R_{25}$. c) The excitation parameter $P$ as a function of galactocentric distance.

than $R^{*}$ results in the wrong $(\mathrm{O} / \mathrm{H})_{R_{23}}$ and $(\mathrm{O} / \mathrm{H})_{P}$ abundances in these $\mathrm{H}_{\text {II }}$ regions and, as consequence, results in the false breaks in the slopes of $(\mathrm{O} / \mathrm{H})_{R_{23}}$ and $(\mathrm{O} / \mathrm{H})_{P}$ abundance distributions. In the case of M101 this problem was clearly noted by Kennicutt \& Garnett (1996). Since they did not use the $R_{23}$ calibration for the outermost $\mathrm{H}$ II regions, their abundance gradient does not show the break.

Around twenty individual measurements of the temperature-sensitive line ratios in $\mathrm{H}_{\text {II }}$ regions of M101 are available now, which makes it possible to determine the oxygen abundance in a number of $\mathrm{H}_{\text {II }}$ regions in $\mathrm{M} 101$ with classic $T_{\mathrm{e}}$-method $\left((\mathrm{O} / \mathrm{H})_{T_{\mathrm{e}}}\right.$ abundances $)$. The $(\mathrm{O} / \mathrm{H})_{T_{\mathrm{e}}}$ data is sufficient in quantity and quality for an accurate determination of the value of the oxygen abundance gradient within M101 (Pilyugin 2001b). The radii interior and exterior to the $R^{*}$ value are sampled. The $(\mathrm{O} / \mathrm{H})_{T_{\mathrm{e}}}$ distribution does not show the flattering at the outer zone of M101 (thick solid line in Figs. 1a,b). This is decisive proof that the breaks in the slopes of $(\mathrm{O} / \mathrm{H})_{R_{23}}$ and $(\mathrm{O} / \mathrm{H})_{P}$ abundance distributions within the disk of M101 is an artifact caused by the wrong oxygen abundances in H II regions with galactocentric distances larger than $R^{*}$.

The break in the slope of the $(\mathrm{O} / \mathrm{H})_{R_{23}}$ abundance gradient in the disk of barred spiral galaxy NGC 1365 was obtained by Roy \& Walsh (1997). Does the break in the slope of the $(\mathrm{O} / \mathrm{H})_{R_{23}}$ abundance gradient in NGC 1365 have the same nature as that in NGC 5457? Around seventy measurements of the strong oxygen lines intensities in $\mathrm{H}_{\text {II }}$ regions in the disk of NGC 1365 are available (Alloin et al. 1981; Pagel et al. 1979; Roy \& Walsh 1997). Figure 2a shows the radial $(\mathrm{O} / \mathrm{H})_{R_{23}}$ abundance distribution (the triangles) for $\mathrm{H}$ II regions in the disk of NGC 1365 derived with Eq. (2). The galactocentric distances are normalized to the isophotal radius $R_{25}$ adopted to be equal to 5.61 arcmin (de Vaucouleurs et al. 1991). Figure 2b shows the radial $(\mathrm{O} / \mathrm{H})_{P}$ abundance distribution (the filled circles) for H II regions in the disk of NGC 1365 derived with Eq. (1). As can be seen in Figs. 2a,b there is break as in the slope of the $(\mathrm{O} / \mathrm{H})_{R_{23}}$ abundance distribution as in the slope of the $(\mathrm{O} / \mathrm{H})_{P}$ abundance distribution at the galactocentric distance $R^{*} \sim 0.7 R_{25}$ although the exact position of the point of break is not beyond question due to large scatter in oxygen abundance at any fixed radius.

Comparison of Figs. 1a and 2 a shows a very important difference between radial abundance distributions in the disk of M101 and in the disk of NGC 1365 . The $(\mathrm{O} / \mathrm{H})_{R_{23}}$ abundance at the point of bend in the disk of NGC 1365 is $12+$ $\log (\mathrm{O} / \mathrm{H})_{R_{23}} \sim 8.6$ and is appreciable higher than the lower boundary $(12+\log (\mathrm{O} / \mathrm{H}) \sim 8.2)$ of the upper branch in the $\mathrm{O} / \mathrm{H}-R_{G}$ diagram while in the case of $\mathrm{M} 101$ the $(\mathrm{O} / \mathrm{H})_{R_{23}}$ abundance at the point of bend is around $12+\log (\mathrm{O} / \mathrm{H})_{R_{23}} \sim 8.3$ and is close to the lower boundary of the upper branch in the $\mathrm{O} / \mathrm{H}$ $R_{G}$ diagram. This high value of $(\mathrm{O} / \mathrm{H})_{R_{23}}$ abundance at the point of bend in the disk of NGC 1365 was a reason why the fact that those $\mathrm{H}_{\text {II }}$ regions belong to the upper branch in the $\mathrm{O} / \mathrm{H}$ $R_{G}$ diagram (and consequently the validity of the $R_{23}$-method) was not doubted in previous studies. However a difference between values of the $(\mathrm{O} / \mathrm{H})_{R_{23}}$ abundances at the point of bend in the disk of M101 and in the disk of NGC 1365 can be easy explained. As it was mentioned above the oxygen abundance derived with the $R_{23}$-method involves a systematic error depending on the excitation parameter $P$ : the less the value of the excitation parameter $P$ the more overestimated the value of oxygen abundance is obtained with the $R_{23}$-method (Pilyugin 2001a,b). In the case of M101 the excitation of the H II regions 
with galactocentric distances around $R^{*}$ is moderate $P \sim 0.7$ (Fig. 1c) and, as a consequence, the $(\mathrm{O} / \mathrm{H})_{R_{23}}$ values in those $\mathrm{H}$ II regions are only slightly overestimated. On the contrary, in the case of NGC 1365 the excitation of the HiI regions with galactocentric distances around $R^{*}$ is low $P \sim 0.3$ (Fig. 2c) and hence the $(\mathrm{O} / \mathrm{H})_{R_{23}}$ values in those $\mathrm{H}$ II regions are significantly overestimated.

At the same time, Fig. 2b shows that the $(\mathrm{O} / \mathrm{H})_{P}$ distribution in the disk of NGC 1365 flattens at the radius where oxygen abundance decreases to around $12+\log (\mathrm{O} / \mathrm{H})=8.2$. This suggests that the breaks in the slopes of $(\mathrm{O} / \mathrm{H})_{P}$ and $(\mathrm{O} / \mathrm{H})_{R_{23}}$ abundance distributions within the disk of NGC 1365 is an artifact caused by the wrong oxygen abundances in $\mathrm{H}$ II regions with galactocentric distances larger than $R^{*}$. Of course, this statement will be an undisputable fact when the low oxygen abundance in the outermost $\mathrm{H}$ II regions in the disk of NGC 1365 will be confirmed by the direct determination with the $T_{\mathrm{e}^{-}}$ method. This statement can be also strengthened by consideration of other galaxies.

We have compiled the published measurements (more than 900 individual measurements) of the strong oxygen lines intensities in $\mathrm{H}_{\text {II }}$ regions in disks of spiral galaxies and derived the radial $(\mathrm{O} / \mathrm{H})_{P}$ abundance distributions. It was found that the oxygen abundances in the disks of a number of galaxies decreases to $12+\log (\mathrm{O} / \mathrm{H})=8.2$ within the isophotal radius. In the disks of ten galaxies the $\mathrm{H}_{\mathrm{II}}$ regions are observed at the radii exterior to the $R^{*}$ (where $R^{*} \sim 0.8 R_{25}$ in NGC 300, $R^{*} \sim 0.5 R_{25}$ in NGC $925, R^{*} \sim 0.7 R_{25}$ in NGC 1365 , $R^{*} \sim 0.9 R_{25}$ in NGC $2805, R^{*} \sim 0.8 R_{25}$ in NGC 3198 , $R^{*} \sim 0.4 R_{25}$ in NGC $3319, R^{*} \sim 0.8 R_{25}$ in NGC 4651, $R^{*} \sim 0.5 R_{25}$ in NGC 5033, $R^{*} \sim 0.6 R_{25}$ in NGC 5457, $R^{*} \sim 0.6 R_{25}$ in NGC 7793). It is remarkable that many galaxies with reported signs of a bend in the slope of the abundance gradient, NGC 300, NGC 5457, NGC 7793 (Vila-Costas \& Edmunds 1992), NGC 3319, NGC 5033 (Zaritsky et al. 1994), NGC 1365 (Roy \& Walsh 1997) are among the galaxies with the $\mathrm{H}$ II regions observed at the radii exterior to the $R^{*}$. If the possibility of a real bend in the slope of the abundance gradient at the radius $R^{*}$ in any given galaxy cannot be conclusively excluded, it is almost beyond belief that a real bend in the slope of abundance gradient takes place at the fixed value of oxygen abundance $12+\log (\mathrm{O} / \mathrm{H})=8.2$ in all galaxies. Thus, the fact that galaxies with signs of a bend in abundance gradient are contained in the list of galaxies with the $\mathrm{H}_{\text {II }}$ regions observed at the radii exterior to the $R^{*}$ can be considered as evidence in favour that the reported bends are false and reasoned by unjustified use of the relationship between oxygen abundance and strong line intensities, which is valid for the $\mathrm{H}$ II regions of the upper branch of the $R_{23}-\mathrm{O} / \mathrm{H}$ diagram only, to the abundance determination in $\mathrm{H}_{\text {II }}$ regions at radii exterior to $R^{*}$, although those $\mathrm{H}$ II regions do not belong to the upper branch of the $R_{23}-$ $\mathrm{O} / \mathrm{H}$ diagram.

Two galaxies NGC 5194 and M81, in which a bend in the slope of the $(\mathrm{O} / \mathrm{H})_{R_{23}}$ abundance gradient was suspected, will be discussed in the next subsection. For the disk of galaxy M33, in which the bend in the slope of $(\mathrm{O} / \mathrm{H})_{R_{23}}$ abundance gradient was reported by Vilchez et al. (1988), the compiled data (40 H II regions) are reasonably well fitted by a single exponential profile.

\subsection{False bends of the "second type"}

The gradient in the excitation parameter $P$ within the disk of M101, Fig. 1c, results in the false increase in the slope of the $(\mathrm{O} / \mathrm{H})_{R_{23}}$ abundance gradient, Fig. 1a. Hence, if there is a bend in the excitation parameter gradient within the disk of a galaxy, one can expect that this results in a false bend in the slope of $(\mathrm{O} / \mathrm{H})_{R_{23}}$ abundance gradient. This effect indeed takes place in the disks of some galaxies. A manifestation of this effect will be demonstrated with a galaxy NGC 2403.

We compiled 47 published measurements of the strong oxygen line intensities in $\mathrm{H}$ II regions of the galaxy NGC 2403 (Fierro et al. 1986; Garnett et al. 1997; Garnett et al. 1999; McCall et al. 1985; Smith 1975; van Zee et al. 1998). Figure 3a shows the radial $(\mathrm{O} / \mathrm{H})_{P}$ abundance distribution (the filled circles) for $\mathrm{H}_{\text {II }}$ regions in NGC 2403 derived with Eq. (1). The galactocentric distances are normalized to the isophotal radius $R_{25}$ which was taken to be equal to 11.45 arcmin (de Vaucouleurs et al. 1991). Figure 3b shows the radial $(\mathrm{O} / \mathrm{H})_{R_{23}}$ abundance distribution (the open circles) for $\mathrm{H}$ II regions in NGC 2403 derived with Eq. (2).

Inspection of Fig. 3a shows that the variation in the logarithm of $(\mathrm{O} / \mathrm{H})_{P}$ abundance with radius can be fitted by a straight line. At the same time the variation in the logarithm of $(\mathrm{O} / \mathrm{H})_{R_{23}}$ abundance with radius shows evidence of a bend in the slope of the abundance gradient, Fig. 3b. The origin of this bend is clearly illustrated in Figs. $3 \mathrm{c}$ and $3 \mathrm{~d}$. Since $(\mathrm{O} / \mathrm{H})_{P}$ abundances are as credible as $(\mathrm{O} / \mathrm{H})_{T_{\mathrm{e}}}$ abundances, the $(\mathrm{O} / \mathrm{H})_{P^{-}}$ $R_{G}$ relation reproduces a real abundance gradient. Then the deviations of $(\mathrm{O} / \mathrm{H})_{R_{23}}$ abundances from the $(\mathrm{O} / \mathrm{H})_{P}-R_{G}$ relation are errors in $(\mathrm{O} / \mathrm{H})_{R_{23}}$ abundances. Inspection of Fig. 3c shows that the average value of errors in $(\mathrm{O} / \mathrm{H})_{R_{23}}$ abundances is minimum at galactocentric distance around $0.5 R_{25}$ and inceases with distance from this point. This behaviour of the average value of errors in $(\mathrm{O} / \mathrm{H})_{R_{23}}$ abundances reflects the behaviour of the average value of the excitation parameter, Fig. 3d; the lower the average value of the excitation parameter the higher the average value of errors in $(\mathrm{O} / \mathrm{H})_{R_{23}}$ abundances.

Thus, a false bend in the slope of the $(\mathrm{O} / \mathrm{H})_{R_{23}}$ abundance gradient can appear due to a bend in the excitation parameter gradient within the disk of a galaxy. The bend in the slope of $(\mathrm{O} / \mathrm{H})_{R_{23}}$ abundance gradients in the disk of NGC 5194 reported by Vila-Costas \& Edmunds (1992) is a manifestation of this effect. This effect is also responsible for the weak break in the slope of the $(\mathrm{O} / \mathrm{H})_{R_{23}}$ abundance gradient in the disk of M81 which was indicated by Zaritsky (1992).

In general, we did not find a meaningful bend in the radial $(\mathrm{O} / \mathrm{H})_{P}$ abundance distributions derived in disks of around 50 spiral galaxies investigated. It should be noted however that the number of observed $\mathrm{H}_{\text {II }}$ regions in half of the considered galaxies is less than 14. Dutil \& Roy (2001) showed that at least $16 \mathrm{H}$ iा regions are needed for a meaningful and robust description of radial abundance gradient in a disk galaxy. If one is looking for a break in the gradient of the spatial distribution, the sample size required is several times larger. Then, the paucity of observational data inhibits the detection of a real bend in the radial $(\mathrm{O} / \mathrm{H})_{P}$ abundance distribution even if this takes place in some galaxies. Thus, we cannot reject the 


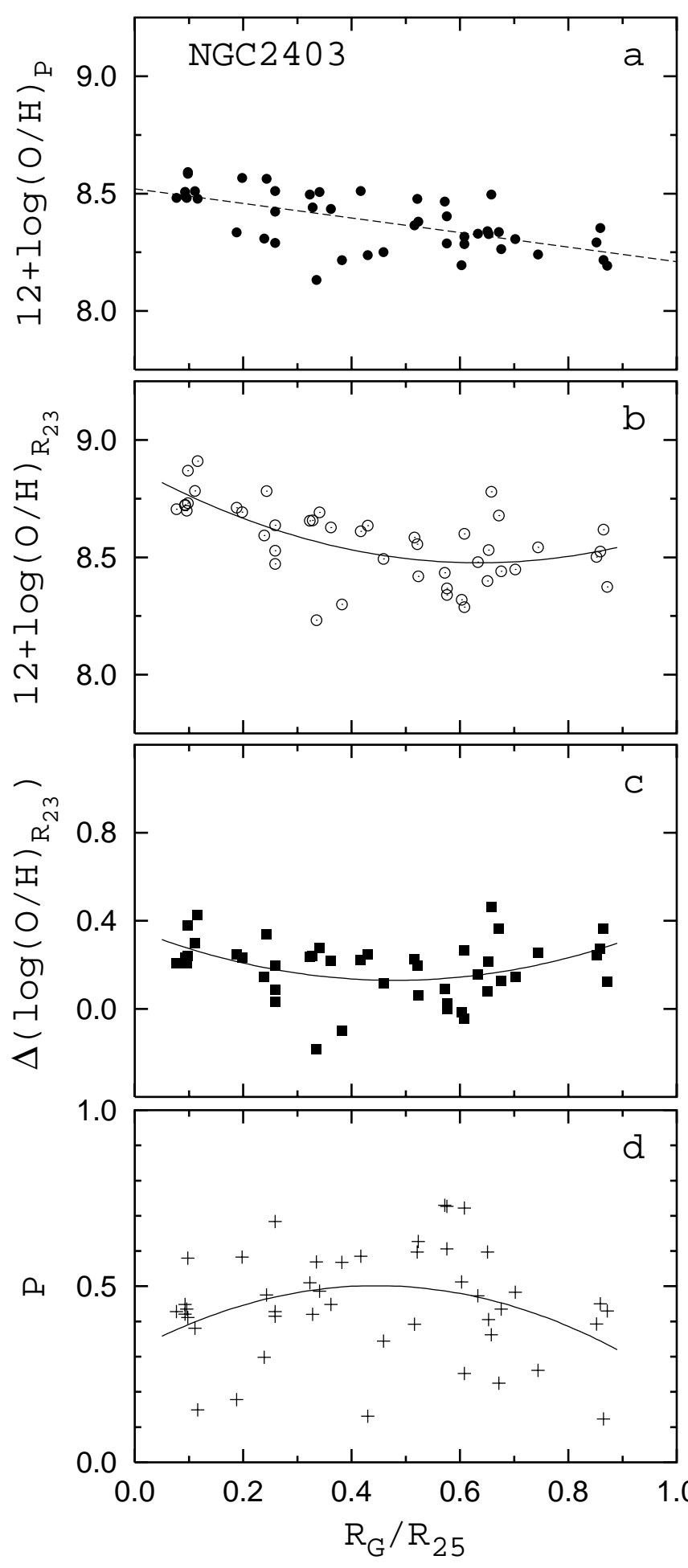

Fig. 3. Gradients in the properties of NGC 2403. The galactocentric distances are normalized to the isophotal radius $R_{25}$. a) The filled circles are $(\mathrm{O} / \mathrm{H})_{P}$ abundances in the $\mathrm{H}_{\text {II }}$ regions, the dashed line is the best fit (the $(\mathrm{O} / \mathrm{H})_{P}-R_{G}$ relation). b) The open circles are $(\mathrm{O} / \mathrm{H})_{R_{23}}$ abundances in $\mathrm{H}_{\text {II }}$ regions, the solid curve is the best fit. c) The squares are the deviations of $(\mathrm{O} / \mathrm{H})_{R_{23}}$ abundances from the $(\mathrm{O} / \mathrm{H})_{P}-R_{G}$ relation. The solid curve is the best fit. d) The pluses are the excitation parameter $P$ in the $\mathrm{H}_{\text {II }}$ regions, the solid curve is the best fit.

existence of a real bend in the radial $(\mathrm{O} / \mathrm{H})_{P}$ abundance distribution in galaxies at all, but we can conclude that at the present time there is no example of a galaxy with an undisputable established bend in the slope of the oxygen abundance gradient.

\section{Conclusions}

Spiral galaxies with a reported bend in the slope of gradient in the $(\mathrm{O} / \mathrm{H})_{R_{23}}$ oxygen abundances (derived with traditionally used $R_{23}$-method) were examined. It was found that the false nature of the reported bends can be easily explained. Two reasons that result in a false bend in the slope of $(\mathrm{O} / \mathrm{H})_{R_{23}}$ gradient are indicated.

First, an unjustified use of the relationship between oxygen abundance and strong line intensities, constructed for the high-metallicity $\mathrm{H}_{\text {II }}$ regions of the upper branch of the $R_{23}$ $\mathrm{O} / \mathrm{H}$ diagram, in the determination of oxygen abundance in low-metallicity $\mathrm{H}$ in regions at the periphery of a galaxy, results in wrong (overestimated) oxygen abundances in those $\mathrm{H}$ II regions, and, as a consequence, false bend in the slope of the abundance gradient appears. A manifestation of this effect has been demonstrated in a convincing way with the galaxy M101. Other galaxies where this effect is expected are listed.

Second, the bend in the excitation parameter gradient within the disk of a galaxy results in a false bend in the slope of the $(\mathrm{O} / \mathrm{H})_{R_{23}}$ gradient since the oxygen abundance derived with the $R_{23}$-method involves a systematic error depending on the excitation parameter. A manifestation of this effect has been demonstrated with the galaxy NGC 2403.

It is concluded that at the present time there is no example of a galaxy with an undisputable established bend in the slope of the oxygen abundance gradient.

Acknowledgements. I thank the anonymous referee for discussion. This study was partly supported by the Joint Research Project between Eastern Europe and Switzerland (SCOPE) No. 7UKPJ62178, the NATO grant PST.CLG.976036, and the Italian national grant delivered by the MURST.

\section{References}

Alloin, D., Edmunds, M. G., Lindblad, P. O., \& Pagel, B. E. J. 1981, A\&A, 101, 377

Castellanos, M., Diáz, A. I., \& Terlevich, E. 2002, MNRAS, 329, 315 de Vaucouleurs, G., de Vaucouleurs, A., Corvin, H. G., et al. 1991, Third Reference Catalog of bright Galaxies (New York: Springer) (RC3)

Dutil, Y., \& Roy, J.-R. 2001, AJ, 122, 1644

Edmunds, M. G., \& Pagel, B. E. J. 1984, MNRAS, 211, 507

Fierro, J., Torres-Peinmert, S., \& Peimbert, M. 1986, PASP, 98, 1032

Friedli, D. 1999, ASP Conf. Ser., in press [astro-ph/9903143]

Friedli, D., Benz, W., \& Kennicutt, R. C. 1994, ApJ, 430, L105

Friedli, D., \& Benz, W. 1995, A\&A, 301, 649

Garnett, D. R., \& Kennicutt, R. C. 1994, ApJ, 426, 123

Garnett, D. R., Shields, G. A., Peimbert, M., et al. 1999, ApJ, 513, 168 Garnett, D. R., Shields, G. A., Skillman, E. D., Sagan, S. P., \& Dufour, R. J. 1997, ApJ, 489, 63

Henry, R. B. C., \& Howard, J. W. 1995, ApJ, 438, 170

Kennicutt, R. C., \& Garnett, D. R. 1996, ApJ, 456, 504

Kinkel, U., \& Rosa, M. R. 1994, A\&A, 282, L37

McCall, M. L., Rybski, P. M., \& Shields, G. A. 1985, ApJSS, 57, 1

Pagel, B. E. J., Edmunds, M. G., Blackwell, D. E., Chun, M. S., \& Smith, G. 1979, MNRAS, 189, 95 
Pilyugin, L. S. 2000, A\&A, 362, 325

Pilyugin, L. S. 2001a, A\&A, 369, 594

Pilyugin, L. S. 2001b, A\&A, 373, 56

Rayo, J. F., Peimbert, M., \& Torres-Peimbert, S. 1982, ApJ, 255, 1

Roy, J.-R., \& Walsh, J. R. 1997, MNRAS, 288, 715

Searle, L. 1971, ApJ, 168, 327

Shields, G. A., \& Searle, L. 1978, ApJ, 222, 821

Smith, H. E. 1975, ApJ, 199, 591
Torres-Peimbert, S., Peimbert, M., \& Fierro, J. 1989, ApJ, 345, 186 van Zee, L., Salzer, J. J., Haynes, M. P., O’Donoghue, A. A., \& Balonek, T. J. 1998, AJ, 116, 2805

Vila-Costas, M. B., \& Edmunds, M. G. 1992, MNRAS, 259, 121

Vilchez, J. M., Pagel, B. E. J., Diaz, A. I., Terlevich, E., \& Edmunds, M. G. 1988, MNRAS, 235, 633

Zaritsky, D. 1992, ApJ, 390, L73

Zaritsky, D., Kennicutt, R. C., Jr., \& Huchra, J. P. 1994, 420, 87 